\title{
Comparación de métodos para la determinación del valor energético de alimentos para rumiantes
}

\section{Comparison of methods to determine the energy value of feeds for ruminants}

\author{
Sandra Posada O, ${ }^{1 *}$ Ph.D, Ricardo Rosero N, ${ }^{1}$ Ph.D, Norberto Rodríguez, ${ }^{2}$ Ph.D, \\ Ana Costa C, ${ }^{2}$ Ph.D.
}

\begin{abstract}
${ }^{1}$ Universidad de Antioquia, Facultad de Ciencias Agrarias, Grupo de Investigación en Ciencias AnimalesGRICA, AA 1226, Medellín, Colombia. 'Universidade Federal de Minas Gerais, Escola de Veterinária, CEP 31270-901, Belo Horizonte, Brasil. *Correspondencia: slposada@agronica.udea.edu.co
\end{abstract}

Recibido: Junio de 2011; Aceptado: Mayo de 2012.

\section{RESUMEN}

Objetivo. Comparar métodos de estimación del TND a partir de ensayos de digestibilidad y análisis químico de la dieta. Materiales y métodos. La digestibilidad in vivo fue determinada usando cebuínos alimentados con heno (Cynodon dactylon L. Pers. cv. Tifton 85), maíz y soya. La digestibilidad in vitro siguió la metodología de Tilley y Terry $(T T)$. Los métodos de estimación del TND fueron: convencional (MET 1), calorimétrico desde valores de combustión cuantificados en bomba calorimétrica (MET 2), calorimétrico desde calores de combustión predefinidos (MET 3), desde la digestibilidad in vivo e in vitro de la materia orgánica (MET 4), desde el modelo multicompartimental del NRC (2001) (MET 5). Los datos se analizaron en diseño completamente aleatorizado y la comparación del efecto promedio se realizó usando la prueba de Tukey $(a=5 \%)$. La intercambiabilidad entre los valores de TND obtenidos desde el procedimiento TT (MET 4) y el MET 5 se valoró a partir del método de Bland-Altman. Resultados. Los valores de TND estimados in vivo por MET 1, MET 3 y MET 4 no presentaron diferencia $(p>0.05)$ pero difirieron del valor obtenido por MET $2(p<0.05)$, que fue menor. Los valores de TND del MET 5 no fueron intercambiables con los obtenidos desde el procedimiento $T$, que superaron los rangos encontrados en la literatura. Conclusiones. La estimación del TND desde MET 2 es más confiable porque no se hacen presunciones u omisiones en el valor energético de los componentes orgánicos. EI MET 5 se mostró promisorio para el cálculo del TND.

Palabras clave: Caloría, calorimetría, digestibilidad in vitro, digestibilidad in vivo (Fuente: Agrovoc). 


\section{ABSTRACT}

Objective. Compare different methods (MET) to calculate TND based on chemical analysis of diets and digestibility trials. Materials and methods. in vivo digestibility trials were determined using zebu animals fed hay (Cynodon dactylon L. Pers. cv. Tifton 85), corn and soybean meal. The in vitro digestibility followed the Tilley and Terry (1963) methodology (TT). Methods for estimating TND were: conventional (MET 1), calorimetric based on the combustion values quantified in a bomb calorimeter (MET 2), calorimetric based on table combustion heat values (MET 3), based on the in vivo e in vitro organic matter digestibility (MET 4), and based on the multi-compartment model by the NRC (2001) (MET 5). Data was analyzed by a completely randomized design and comparison of the average effect was conducted using Tukey test $(a=5 \%)$. The Bland-Altman method was used to evaluate the exchangeability between TND values obtained from $T$ assay (MET 4) and MET 5 . Results. The TND values estimated in vivo by MET 1, MET 3 y MET 4 showed no statistical difference $(p>0.05)$ but differed from the value obtained by MET $2(p<0.05)$, which was lower. TND values estimated by MET 5 were not exchangeable with those obtained by $\Pi T$ assay, which were above the ranges established by literature. Conclusions. TND estimate by MET 2 proved to be more reliable, because it did not include assumptions or omissions for energy values of the organic components. MET 5 proved to be promising for the calculation of TND.

Key words: Calorie, calorimetry, in vitro digestibility, in vivo digestibility (Source: Agrovoc).

\section{INTRODUCCIÓN}

El análisis químico no es suficiente para conocer el valor nutritivo de los alimentos, siendo también importante considerar los procesos asociados con su digestión, absorción y metabolismo. Las pruebas de digestibilidad permiten estimar la proporción de nutrientes presentes en una ración que puedan ser absorbidos por el aparato digestivo del animal y que, por tanto, no se eliminan con las heces (1).

La información sobre el potencial energético de los alimentos es fundamental para su valoración nutricional y económica. Si bien la energía bruta puede ser medida de forma simple a través del empleo de una bomba calorimétrica, su validez en nutrición animal es cuestionable por la variabilidad que registran los alimentos en digestibilidad y metabolismo. Entendiendo que las pérdidas fecales son las más representativas de la energía consumida, ensayos de digestibilidad que permitan cuantificar su magnitud estarán dando una mejor aproximación de la energía realmente disponible para el animal. Factores dependientes del alimento y del animal afectan la digestibilidad del alimento y, por tanto, su valor energético. Entre los primeros se tiene la composición del alimento consumido (contenido de material celulósico y graso, concentración mineral), el tratamiento al que ha sido sometido (secado, molienda) y el efecto asociativo entre los alimentos que componen la ración (1). Los factores animales incluyen el nivel de consumo, la capacidad de selección del animal en función de la oferta de material, la disponibilidad de agua, la tasa de pasaje del alimento, la eficiencia metabólica de los animales y las condiciones ambientales (temperatura, humedad relativa) en las que se encuentren (2).

Para la determinación del coeficiente de digestibilidad se han empleado diversos métodos in vivo e in vitro. Entre los procedimientos in vivo, la colección total de heces es el método más confiable por involucrar directamente factores del alimento y del animal que afectan el aprovechamiento nutricional, no obstante, es laborioso, costoso, demanda tiempo e infraestructura e implica algunas restricciones al manejo ordinario de animales en producción (2). Dadas estas restricciones, los métodos de digestibilidad in vitro representan una alternativa de trabajo por su rapidez y confiabilidad, siempre que se simulen adecuadamente los procesos de digestión (3). Métodos de estimación de la energía disponible que integran el análisis químico de los alimentos con modelos matemáticos unicompartimentales y multicompartimentales también han sido reportados por la literatura $(4,5)$.

El total de nutrientes digestibles (TND), como unidad de expresión del contenido energético de los alimentos, constituye una medida aproximada de la digestibilidad (6) y cuando su valor es conocido otras expresiones de energía pueden ser calculadas mediante el uso de ecuaciones apropiadas (5). El objetivo de este trabajo fue presentar y comparar varios métodos de estimación del TND a partir de ensayos de digestibilidad in vivo, in vitro y a partir modelos matemáticos que incorporan el análisis químico de la ración. 


\section{MATERIALES Y MÉTODOS}

Tipo de estudio. Los datos de este trabajo se generaron de un experimento con ganado Nellore realizado durante cuatro períodos diferentes, correspondientes a un intervalo promedio de peso de $81.43 \mathrm{Kg}$. Durante cada período se realizaron pruebas de digestibilidad in vivo. La prueba de digestibilidad in vitro, incluyendo todos los alimentos garantizados durante el trabajo, se realizó al final de la fase experimental para garantizar idénticas condiciones de laboratorio.

Sitio de estudio. El experimento se realizó en la Escuela de Veterinaria de la Universidad Federal de Minas Gerais, Belo Horizonte (Brasil). La ciudad de Belo Horizonte está situada a $900 \mathrm{msnm}$, posee una temperatura media de $23^{\circ} \mathrm{C}$, humedad relativa del $65 \%$ y precipitación promedio anual de $1600 \mathrm{~mm}$, tratándose de un clima tropical de altitud según la clasificación de Köppen (7). Geográficamente se encuentra a $19^{\circ} 55^{\prime} 15^{\prime \prime}$ de latitud Sur y $43^{\circ} 56^{\prime} 16^{\prime \prime}$ de longitud occidente, con respecto al meridiano de Greenwich.

Animales e instalaciones experimentales. Cinco cebuínos enteros de raza Nellore, con peso vivo medio inicial de $200 \mathrm{Kg}$, fueron mantenidos en régimen de confinamiento en galpón cubierto, en corrales individuales $(2 \times 1.5 \mathrm{~m})$, con piso de concreto revestido de tapete, comederos y bebederos individuales.

Alimentación. La dieta estuvo constituida por heno de Tifton 85 (Cynodon dactylon L. Pers. cv. Tifton 85), suplemento concentrado a base de maíz y torta de soya y sal mineralizada comercial. La proporción forraje-concentrado varió en función de la edad de los animales, buscando una composición nutricional que permitiera obtener ganancias medias próximas a $700 \mathrm{~g} / a n i m a l / d i ́ a$, de acuerdo con las recomendaciones de Valadares et al (8) (Tabla 1). La ganancia media de peso obtenida fue $1058.25 \mathrm{~g} /$ animal/día.

La ración fue suministrada ad libitum dos veces al día, a las 8 y 17 horas, en la forma de ración total mezclada. La cantidad de alimento fue diariamente ajustada permitiendo sobrantes entre 5 y $10 \%$ de lo ofrecido, las cuales fueron pesadas y muestreadas diariamente para análisis de materia seca. El consumo de
Tabla 1. Composición porcentual y descripción química de la ración, expresadas como porcentaje de la materia seca.

\begin{tabular}{lcccc}
\hline \multirow{2}{*}{ Ingred. } & \multicolumn{4}{c}{ Período } \\
\cline { 2 - 5 } & $\mathbf{1}$ & $\mathbf{2}$ & $\mathbf{3}$ & $\mathbf{4}$ \\
\hline $\begin{array}{l}\text { Heno (Cynodon } \\
\text { dactylon (L.) Pers. }\end{array}$ & 60 & 60 & 70 & 80 \\
$\begin{array}{l}\text { CV. Tifton 85) } \\
\text { Maíz }\end{array}$ & 20 & 25 & 18 & 10 \\
Torta de soya & 20 & 15 & 12 & 10 \\
\hline Composición química ${ }^{2}$ & & & \\
\hline MS & 89.73 & 88.87 & 89.62 & 90.11 \\
PB & 14.49 & 15.13 & 11.82 & 14.45 \\
EE & 1.58 & 2.40 & 2.17 & 2.06 \\
MI & 5.42 & 5.80 & 6.47 & 6.37 \\
FDN ${ }_{p}$ & 55.51 & 48.40 & 56.72 & 65.08 \\
FDA & 27.31 & 22.15 & 27.68 & 32.09 \\
Lignina $_{\text {CHO }}$ & 3.13 & 1.90 & 2.84 & 2.85 \\
CNE & 78.52 & 76.67 & 79.54 & 77.12 \\
EB (Kcal/Kg MS) & 23.01 & 28.27 & 22.82 & 12.03 \\
\hline
\end{tabular}

Ingred. = Ingredientes ${ }^{1}$

${ }^{1}$ Balances minerales, de acuerdo con las recomendaciones de Valadares y col. [19], determinaron la cantidad de sal mineralizada garantizada. Composición por kilogramo: $160 \mathrm{~g}$ calcio, $60 \mathrm{~g}$ fósforo, $110 \mathrm{~g}$ sodio, 10 g magnesio, $50 \mathrm{~g}$ azufre, $82 \mathrm{mg}$ cobalto, $800 \mathrm{mg}$ cobre, $120 \mathrm{mg}$ yodo, $3600 \mathrm{mg}$ manganeso, $27 \mathrm{mg}$ selenio, $5200 \mathrm{mg}$ zinc, $4700 \mathrm{mg}$ hierro, 600 mg (máx) de flúor

${ }^{2} \mathrm{MS}=$ materia seca, $\mathrm{PB}=$ proteína bruta, $\mathrm{EE}=$ extracto etéreo, $\mathrm{MI}=$ material inorgánico, $\mathrm{FDN}_{\mathrm{p}}=$ fibra en detergente neutro corregida para proteína, $\mathrm{FDA}=$ fibra en detergente ácido, $\mathrm{CHO}_{\mathrm{t}}=$ carbohidratos totales, $\mathrm{CNE}=$ carbohidratos no estructurales, $\mathrm{EB}=$ energía bruta

materia seca fue obtenido por la diferencia entre la cantidad de alimento ofrecido y el sobrante. La ración fue la misma para todos los animales, variando sólo las cantidades garantizadas de acuerdo con el manejo previamente expuesto.

Ensayos de digestibilidad aparente in vivo. Las pruebas de digestibilidad tuvieron una duración de cinco días y fueron realizadas durante los cuatro períodos evaluados. La producción de materia seca fecal fue determinada por el procedimiento tradicional de colecta total y la composición química de las heces fue valorada desde colecta fecal individual realizada diariamente a mañana y tarde. Porciones de aproximadamente $250 \mathrm{~g}$ de heces fueron colectadas directamente del ano, identificadas y congeladas $a-10^{\circ} \mathrm{C}$. Al final del período de colecta, las heces fueron sometidas a presecado en estufa de ventilación forzada a $65^{\circ} \mathrm{C}$ por 96 horas y después de pasar por un molino estacionario "Thomas-Wiley", adaptado con tamiz de $1 \mathrm{~mm}$, se realizó una muestra compuesta por animal, en proporción con la excreción de materia seca fecal determinada diariamente. Este material fue conservado en frascos plásticos herméticamente cerrados para su posterior análisis químico. 
Ensayo de digestibilidad in vitro. La digestibilidad in vitro de los alimentos individuales, de la mezcla concentrada y de la ración total mezclada fue determinada en un fermentador ruminal DAISY ${ }_{\text {II }}\left(\right.$ ANKOM $\left.^{\circledR}\right)$, conservando la proporción en la cual participaron en la dieta ofrecida a los animales. La metodología desarrollada correspondió a la propuesta realizada por Tilley y Terry (9). La preparación del medio de cultivo y del inóculo, así como la determinación de la digestibilidad de la materia seca se basó en el protocolo propuesto por el proveedor del fermentador (10).

Análisis químico. En los alimentos, los sobrantes y las heces se realizó análisis de materia seca (MS), proteína bruta (PB), extracto etéreo (EE), cenizas (MI) (11), energía bruta (EB) (12) y fibra en detergente neutro (FDN) (13) corregida para proteína $\left(F_{p N}\right)(14)$. Los carbohidratos totales fueron obtenidos por la ecuación $\mathrm{CHO}_{\mathrm{t}}=$ 100-(\%PB+\%EE+\%MI), y los carbohidratos no estructurales (CNE) por la diferencia $\mathrm{CHO}_{\mathrm{t}}{ }^{-}$ $\mathrm{FDN}_{\mathrm{p}}$ (15). El análisis de fibra en detergente ácido (FDA) (13) y de lignina (16) se efectuó únicamente en los alimentos. En los residuos de incubación in vitro sólo se determinó el contenido de MS y de MI.

Determinación del total de nutrientes digestibles (TND). El contenido de TND de los alimentos fue determinado a través de cinco métodos (MET) diferentes:

Por el método convencional (MET 1). EI TND fue estimado a partir de la ecuación:

$\operatorname{TND}(\%)=(P B D+C N E D+F D N$ D + $2.25 * \mathrm{EED}) / 100$, donde $\mathrm{PBD}=$ proteína bruta digestible, CNED = carbohidratos no estructurales digestibles, $F D N_{p} D=$ fibra en detergente neutro digestible corregida para proteína y EED = extracto etéreo digestible (1).

Por el método calorimétrico a partir de valores de combustión cuantificados directamente en bomba calorimétrica (MET2). (17). LoS coeficientes de digestibilidad de la energía fueron multiplicados por un factor de ajuste $(F)$, calculado como $\mathrm{F}=(\mathrm{MO} / 100) *(100+(\mathrm{EE} * 2.25)-\mathrm{EE}) / 100$, donde $\mathrm{MO}=$ porcentaje de materia orgánica en la ración y $\mathrm{EE}=$ porcentaje de $\mathrm{EE}$ en la materia orgánica. Los coeficientes de digestibilidad de la energía correspondieron a la energía digestible (ED), expresada como porcentaje del contenido de energía bruta (EB), y fueron determinados a partir de la cuantificación del contenido de EB en alimentos, los sobrantes y las heces, mediante el uso de la bomba calorimétrica.

Por el método calorimétrico a partir de calores de combustión predefinidos, sin empleo de la bomba calorimétrica (MET 3). (17). Partiendo del mismo procedimiento indicado en el MET 2, la única variante consistió en la estimación del contenido de EB de los alimentos, los sobrantes y las heces usando calores de combustión predefinidos para los nutrientes orgánicos, sin empleo directo de la

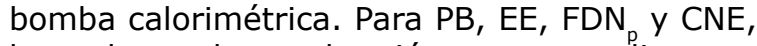
los calores de combustión correspondieron a $5.65,9.40,4.25$ y $4.15 \mathrm{Kcal} / \mathrm{g}$, respectivamente (17).

\begin{abstract}
A partir de la digestibilidad de la materia orgánica (MET 4). (18). El coeficiente de digestibilidad de la materia orgánica (MO) fue multiplicado por el factor de ajuste $\mathrm{F}$ previamente descrito en el MET 2.

Por medio del modelo multicompartimental NRC (MET 5). (4). A partir de la descripción química de los alimentos, el TND fue calculado como TND ${ }_{1 \times}(\%)=d v C N E+\operatorname{dvPB}+(\operatorname{dvAG} * 2.25)$ + dvFDN -7 , siendo 7 el valor correspondiente a las pérdidas metabólicas fecales y dv la digestibilidad verdadera de cada una de las fracciones, que fue encontrada desde las siguientes ecuaciones:
\end{abstract}

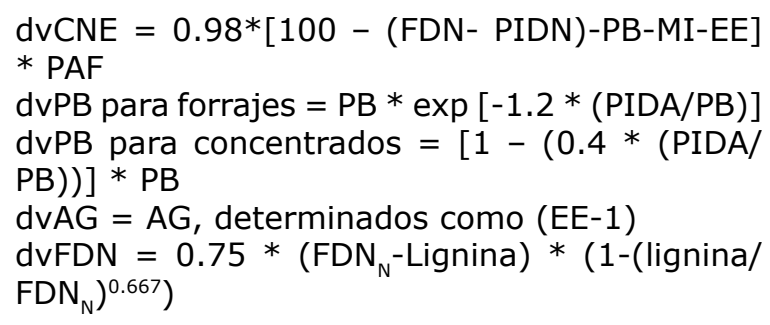

Donde PIND y PIDA= proteína insoluble en detergente neutro y ácido, respectivamente; $\mathrm{FDN}_{\mathrm{N}}=\mathrm{FDN}$ libre de nitrógeno, $\mathrm{PAF}=$ factor de ajuste por procesamiento, correspondiente a 1 para grano molido. Todas las variables contempladas en la ecuación estuvieron expresadas como porcentaje de la materia seca.

A partir de la digestibilidad in vivo de la ración total fue calculado el TND conforme los cuatro primeros métodos (MET 1, MET 2, MET 3 y MET 4). Desde el análisis in vitro de los alimentos, esto es, ensayo de Tilley y Terry (9) y descripción química incorporada al modelo multicompartimental del NRC (4), la determinación del TND (\%) se realizó conforme MET 4 y MET 5, respectivamente. 
Estimación del valor calórico del TND. El valor calórico (VC) del TND estimado por los diferentes métodos, con base en los resultados de digestibilidad in vivo, fue calculado a partir de la relación propuesta por Crampton et al (19): $\mathrm{VC}($ Mcal ED/Kg TND) = [ED del alimento (Mcal/ $\mathrm{Kg} \mathrm{MS}) / \mathrm{TND}(\%)] * 100$

Análisis estadístico. Bajo un diseño de clasificación experimental completamente aleatorizado, balanceado, efecto fijo, se valoró el efecto del método de estimación sobre los valores de TND (\%) obtenidos a partir de las pruebas de digestibilidad in vivo y su correspondiente valor calórico. La comparación del efecto promedio de los métodos se realizó por medio de la prueba de Tukey al $5 \%$ de significancia. Este procedimiento se complementó con un análisis de estadística descriptiva, incluyendo media y desviación estándar.

El grado de intercambiabilidad entre los valores de TND (\%) obtenidos desde el ensayo de Tilley y Terry (9) (estimación del TND a partir de la MET 4) y el modelo multicompartimental del NRC (4) (estimación del TND a partir de la MET 5) se valoró a partir del método de Bland-Altman (20). Este método incluyó la construcción de un gráfico de la media de medidas pareadas (eje $x$ ) contra sus diferencias (eje $y$ ). El intervalo de confianza alrededor de la media de las diferencias fue calculado (media de las diferencias \pm 1.96 $x$ desviación estándar de las diferencias) y posteriormente se efectúo análisis de regresión para las diferencias $(y)$ y los promedios $(x)$ entre ambas metodologías, con el fin de establecer si existió significancia estadística de la pendiente. Para el análisis de los datos fueron usados los procedimientos PROC GLM, PROC MEANS y PROC REG del paquete estadístico SAS (21).

\section{RESULTADOS}

La digestibilidad aparente de la materia seca y de las diversas fracciones nutricionales, resultante de los ensayos de digestibilidad in vivo realizados en los diferentes períodos experimentales, se presenta en la tabla 2.

El promedio de TND (\%) estimado para la ración total mezclada conforme los diferentes métodos, a partir de los resultados de digestibilidad in vivo, y su respectivo valor calórico se presenta en la tabla 3. Los valores de TND y su equivalente calórico no presentaron diferencia significativa $(p>0.05)$ cuando fueron estimados por MET 1, MET 3 y MET 4 ; no obstante, los tres métodos anteriores se mostraron estadísticamente diferentes $(p<0.05)$ del MET 2, que registró menor TND y superior valor calórico. Los coeficientes de variación del TND para MET 1, MET 2, MET 3 y MET 4, fueron $4.43,3.94,4.36$ y 4.50 , respectivamente. Para estos mismos métodos, la dispersión porcentual en el valor calórico fue $3.80,2.20,3.72$ y 3.80 , correspondientemente (Tabla 3).

EI porcentaje de MOD determinado por el procedimiento de Tilley y Terry (9), así como el contenido de TND predicho desde esa fracción (MET 4) y desde el modelo multicompartimental del NRC (4) (MET 5) se muestran en la tabla

Tabla 2. Porcentajes de digestibilidad aparente in vivo de diversos constituyentes nutricionales en los cuatro períodos experimentales

\begin{tabular}{|c|c|c|c|c|c|c|c|c|}
\hline \multirow{3}{*}{ Nutriente } & \multicolumn{8}{|c|}{ Período } \\
\hline & \multicolumn{2}{|c|}{1} & \multicolumn{2}{|c|}{2} & \multicolumn{2}{|c|}{3} & \multicolumn{2}{|c|}{4} \\
\hline & Prom $^{1}$ & $\mathrm{DE}^{\mathbf{1}}$ & Prom & DE & Prom & DE & Prom & DE \\
\hline MS & 67.22 & 2.59 & 71.05 & 1.53 & 71.16 & 2.23 & 74.48 & 2.63 \\
\hline MO & 68.52 & 2.44 & 72.17 & 1.59 & 72.53 & 2.22 & 75.75 & 2.58 \\
\hline PB & 75.68 & 3.65 & 76.62 & 0.70 & 74.11 & 2.78 & 78.32 & 1.18 \\
\hline $\mathrm{EE}$ & 64.92 & 2.93 & 75.57 & 0.95 & 74.19 & 2.71 & 62.72 & 5.72 \\
\hline $\mathrm{FDN}_{\mathrm{p}}$ & 58.20 & 3.36 & 62.20 & 1.78 & 68.20 & 1.97 & 77.33 & 2.48 \\
\hline CNE & 87.39 & 1.03 & 85.20 & 2.31 & 82.02 & 2.61 & 66.40 & 5.59 \\
\hline Energía & 65.78 & 2.70 & 67.68 & 1.70 & 67.55 & 2.59 & 66.16 & 3.41 \\
\hline
\end{tabular}

${ }_{1}^{1}$ Prom = promedio; DE = desviación estándar

Tabla 3. Porcentaje de total de nutrientes digestibles (TND) estimado por diferentes métodos a partir de resultados de digestibilidad in vivo y su correspondiente valor calórico (VC).

\begin{tabular}{|c|c|c|c|c|c|c|c|c|}
\hline & \multicolumn{8}{|c|}{ Método } \\
\hline & \multicolumn{2}{|c|}{1} & \multicolumn{2}{|c|}{2} & \multicolumn{2}{|c|}{3} & \multicolumn{2}{|c|}{4} \\
\hline & Prom $^{2}$ & $\mathbf{D E}^{2}$ & Prom & DE & Prom & DE & Prom & DE \\
\hline $\mathrm{TND}^{1}, \%$ & $69.76 a$ & 3.09 & $64.56 b$ & 2.54 & $69.89 a$ & 3.05 & $69.82 a$ & 3.14 \\
\hline VC, Mcal ED/Kg TND & $3.82 b$ & 0.15 & $4.12 \mathrm{a}$ & 0.09 & $3.81 \mathrm{~b}$ & 0.14 & $3.82 \mathrm{~b}$ & 0.14 \\
\hline
\end{tabular}

${ }^{1}$ Dentro de una fila, promedios sin letra común presentan diferencia estadística $(p<0.05)$

${ }^{2}$ Prom = promedio; DE = desviación estándar 
4. Los mayores coeficientes de variación del porcentaje de TND se registraron para el heno y la ración total mezclada, siendo superiores cuando se trató del ensayo de Tilley y Terry (9). A través de este ensayo, el coeficiente de variación del TND fue de $17.42,15.97,1.43,2.95$ y $2.15 \%$ para la ración total mezclada, el heno, el maíz, la torta de soya y el suplemento concentrado, respectivamente. Para los mismos alimentos, la dispersión del TND cuando se estimó por el modelo multicompartimental (4) fue 4.91, 5.14, $2.22,3.11$ y $1.79 \%$, correspondientemente.

Tabla 4. Porcentaje de materia orgánica digestible (MOD) y del total de nutrientes digestibles (TND) determinados desde ensayo de digestibilidad in vitro de Tilley y Terry (1963) y modelo multicompartimental del NRC (2001)

\begin{tabular}{|c|c|c|c|c|}
\hline \multirow[t]{2}{*}{ Alimento } & \multirow[t]{2}{*}{ Período } & \multicolumn{2}{|c|}{$\begin{array}{c}\text { Procedimiento } \\
\text { Tilley y Terry } \\
\text { (1963) }\end{array}$} & \multirow{2}{*}{$\begin{array}{c}\begin{array}{c}\text { Modelo } \\
\text { NRC } \\
(2001)\end{array} \\
\% \text { TND }\end{array}$} \\
\hline & & $\begin{array}{l}\% \\
\text { MOD }\end{array}$ & \%TND & \\
\hline \multirow{6}{*}{$\begin{array}{l}\text { Heno (Cynodon dactylon } \\
\text { (L.) Pers. cv. Tifton 85) }\end{array}$} & 1 & 41.59 & 39.62 & 52.76 \\
\hline & 2 & 55.30 & 53.13 & 59.76 \\
\hline & 3 & 58.98 & 56.48 & 56.91 \\
\hline & 4 & 46.60 & 44.54 & 55.79 \\
\hline & Prom & 50.62 & 48.44 & 56.31 \\
\hline & $D E$ & 7.95 & 7.74 & 2.89 \\
\hline \multirow[t]{6}{*}{ Maíz } & 1 & 92.91 & 94.24 & 85.64 \\
\hline & 2 & 88.83 & 91.49 & 87.71 \\
\hline & 3 & 90.51 & 91.48 & 85.21 \\
\hline & 4 & 89.46 & 92.94 & 89.39 \\
\hline & Prom & 90.43 & 92.54 & 86.99 \\
\hline & $D E$ & 1.79 & 1.33 & 1.93 \\
\hline \multirow[t]{6}{*}{ Torta de soya } & 1 & 95.48 & 91.25 & 79.00 \\
\hline & 2 & 96.26 & 88.52 & 76.95 \\
\hline & 3 & 92.87 & 85.28 & 73.32 \\
\hline & 4 & 92.65 & 86.62 & 77.20 \\
\hline & Prom & 94.32 & 87.92 & 76.62 \\
\hline & $D E$ & 1.83 & 2.59 & 2.38 \\
\hline & 1 & 91.57 & 90.20 & 82.32 \\
\hline & 2 & 92.29 & 91.24 & 83.63 \\
\hline $\begin{array}{c}(\text { maíz-soya })^{1} \\
50-50\end{array}$ & 3 & 89.69 & 87.33 & 80.34 \\
\hline \multirow{2}{*}{$\begin{array}{l}62.5-37.5 \\
60-40\end{array}$} & 4 & 88.81 & 87.65 & 83.29 \\
\hline & Prom & 90.59 & 89.11 & 82.39 \\
\hline $50-50$ & $D E$ & 1.61 & 1.92 & 1.48 \\
\hline Ración total mezclada & 1 & 59.42 & 57.38 & 64.58 \\
\hline$\left(\right.$ heno, maíz, soya) ${ }^{2}$ & 2 & 65.51 & 63.67 & 69.56 \\
\hline $6-20-20$ & 3 & 58.04 & 55.86 & 64.19 \\
\hline $60-25-15$ & 4 & 42.84 & 41.21 & 61.99 \\
\hline $70-18-12$ & Prom & 56.45 & 54.53 & 65.08 \\
\hline $80-10-10$ & $D E$ & 9.64 & 9.50 & 3.20 \\
\hline
\end{tabular}

${ }^{1}$ Alimentos mezclados de acuerdo a su participación porcentual en la elaboración del suplemento concentrado

${ }^{2}$ Alimentos mezclados de acuerdo a su participación porcentual en la ración total mezclada ofrecida a los animales

A partir de los valores presentados en la tabla 4, fue realizada la prueba de intercambiabilidad de Bland-Altman, basada en el análisis de regresión para las diferencias $(y)$ y los promedios $(x)$ entre los valores de TND estimados desde el ensayo de Tilley y Terry (9) y aquellos obtenidos desde el modelo multicompartimental del NRC (4) (Figura 1). La pendiente de regresión fue estadísticamente significativa $(p=0.0001)$ y el alto grado de dispersión en las diferencias generó un intervalo de confianza (o límite de concordancia) comprendido entre -17.80 y 19.86 .

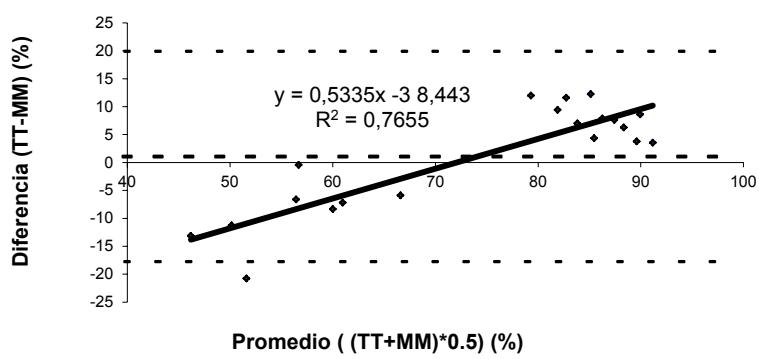

Figura 1. Análisis de Bland-Altman para el TND estimado desde el análisis in vitro de los alimentos ( $\mathrm{T}=$ ensayo de Tilley y Terry, 1963; $\mathrm{MM}=$ modelo multicompartimental del NRC, 2001).

\section{DISCUSIÓN}

Si bien el TND ha sido ampliamente aceptado en circunstancias prácticas de alimentación, se trata de un sistema empírico de valoración energética, donde los valores asignados a los alimentos pueden carecer de precisión dependiendo del método de estimación. En el presente estudio, el TND obtenido para la ración total mezclada, luego de aplicar los resultados derivados del ensayo de digestibilidad in vivo, no presentó diferencia estadística significativa $(p>0.05)$ entre tres de los cuatro métodos de estimación propuestos, registrando un valor medio de $69.82 \%$ (Tabla 3 ). Los tres métodos en mención, correspondientes a MET 1, MET 3 y MET 4, se basaron en el análisis proximal, característica común que puede estar explicando la similitud en los valores de TND obtenidos y que permite clasificarlos como métodos de estimación indirectos, que llevan implícita la falta de precisión asociada con la valoración química. EI MET 2, que presentó diferencia estadística $(p<0.05)$ con los anteriores métodos, superó esta limitante por usar directamente los valores de energía bruta obtenidos de la combustión del alimento, los sobrantes y las heces en la bomba calorimétrica, generando información sobre la energía digestible (luego de aplicar la relación EB absorbida/EB consumida), que luego fue transformada hasta TND. Este método, además, sólo requiere de la determinación de las cenizas y el extracto etéreo en los alimentos evaluados.

El valor medio obtenido con el MET 2 (64.56\%) (Tabla 3), aunque inferior en aproximadamente $8 \%$ respecto los demás métodos evaluados (media de $69.82 \%$ ), no es cuestionable y fue considerado el valor referencia para el presente trabajo, toda vez que la bomba calorimétrica concede a los diversos constituyentes orgánicos su verdadero valor energético, tanto para el alimento consumido como para la fracción digestible, a diferencia de lo que ocurre en el cálculo del TND por el método convencional (MET 1), donde solamente el valor 
calórico extra de las grasas es considerado para la fracción digerida, pero no para la fracción consumida, última fracción donde se considera que la grasa tiene un valor energético equivalente a los carbohidratos y las proteínas. Además, el TND determinado por el método convencional presenta también la limitante de omitir el calor de combustión adicional que tienen las proteínas en relación con los carbohidratos, 5.65 vs. 4.20 $\mathrm{Kcal} / \mathrm{g}$, respectivamente, asumiendo que todas las proteínas y carbohidratos consumidos y digestibles tienen igual valor energético y que todas las grasas poseen un valor energético 2.25 veces mayor que los carbohidratos y las proteínas, supuestos que conllevan a inadecuada evaluación de un alimento o ración.

Según Lofgreen (18), aunque la composición de la materia orgánica del alimento y de las heces es incomparable por las diferencias existentes en la digestibilidad de los nutrientes, los calores de combustión de ambos materiales no son tan desiguales, lo que hace posible estimar el contenido de TND desde el coeficiente de digestión de la materia orgánica. Los resultados del presente trabajo no validaron esta premisa, porque de acuerdo con el planteamiento del autor era de esperarse que el coeficiente de digestión de la MO, que registró un promedio de $72.24 \%$ para los cuatro períodos experimentales, no fuera tan diferente del coeficiente de digestión promedio de la energía, 66.79\% (para el mismo intervalo de tiempo) (Tabla 2), caso en el cual se obtendrían valores de TND similares a partir del MET 2 y del MET 4. Se resalta que la diferencia estadística $(p<0.05)$ entre los métodos 2 y 4 (Tabla 3$)$, que emplearon el contenido de ED y de MOD para estimar el porcentaje de TND aplicando el factor de ajuste $F$ sugerido por Lofgreen $(17,18)$, no fue determinada por la utilización de este factor, ya que el mismo osciló entre 0.96 y 0.97 , afectando en igual medida los valores iniciales de ED y MOD.

Independientemente de las ventajas o desventajas asociadas con los métodos de estimación del TND evaluados, se destacan los bajos coeficientes de variación registrados (Tabla 3), indicando la consistencia en los resultados derivados de los ensayos de digestibilidad in vivo (de la ración total mezclada) (Tabla 2). Situación contraria se registró con el ensayo in vitro de Tilley y Terry (9), donde la media de TND para la ración total mezclada presentó un coeficiente de variación superior (Tabla 4).

En concepto de Lofgreen (17), algunas de las incertidumbres de la medición del TND deben superarse por medir la ED, siendo importante el establecimiento de la relación ED/TND por la repercusión práctica que los valores de TND cobran en los estándares de alimentación. Factores de conversión derivados de comparar valores de TND y ED determinados en una misma prueba de digestión resultarán más significativos que un factor calculado teórico (22).

En el presente trabajo, el valor calórico del TND (de la ración total mezclada durante los ensayos in vivo) fue de $3.82,4.12,3.81$ y $3.82 \mathrm{Mcal} / \mathrm{Kg}$ TND para los métodos de estimación 1, 2, 3 y 4, respectivamente (Tabla 3), mostrándose inferior a $4.409 \mathrm{Mcal}$ ED/Kg TND, reportado por el NRC (23). Debido a que los nutrientes tienen diferentes calores de combustión, el valor calórico del TND no es constante entre alimentos. De acuerdo con el NRC (4), cuando una alta proporción del TND de un alimento se deriva de la proteína, el valor calórico del TND supera las 4.409 Mcal; no obstante, cuando una alta proporción de su TND es provista por carbohidratos o grasa, la equivalencia energética será inferior a 4.409 Mcal/Kg TND. El valor calórico encontrado en este trabajo puede explicarse entonces por la composición de la ración, donde los carbohidratos totales constituyeron en promedio $77.96 \%$ de la ración ofrecida (Tabla 1). El extracto etéreo sólo correspondió al $2.05 \%$ de lo ofrecido, razón por la cual su participación en el cálculo del TND no fue representativa. El superior valor calórico registrado para el MET 2, se corresponde con el menor porcentaje de TND estimado por este método.

Cuando se compararon los métodos de estimación del TND a partir de procedimientos in vitro (MET 4 y MET 5) se observó que los mismos condujeron a sobre o subvaloración de la digestibilidad en función del sustrato evaluado (Tabla 4). En este sentido, la significancia estadística de la pendiente de regresión en la prueba de intercambiabilidad de Bland-Altman (Figura 1) indicó que la discrepancia entre el valor de TND estimado desde el ensayo de Tilley y Terry (9) (MET 4) y aquel obtenido desde la aplicación del modelo multicompartimental del NRC (4) (MET 5) no se mantuvo constante en todo el rango o intervalo de la distribución, sino que incrementó con el aumento de los valores promedio de TND. Tratándose del heno, cuyo valor promedio de TND para ambos métodos de estimación fluctuó entre 46.19 (en el período 1) y $56.59 \%$ (en el período 3), o de la ración total, cuyo media de TND varió entre 51.60 (en el período 4) y 66.62\% (en el período 2 ) (Tabla 4), las diferencias siempre estuvieron a favor del MET 5, con los puntos ubicados por debajo del valor cero en el eje de las abscisas en el análisis de intercambiabilidad (Figura 1). Para los componentes del suplemento, a saber, maíz y torta de soya, el promedio de TND estimado por los dos métodos se mantuvo entre 79.30 (para la torta de soya en el período 3 ) y $91.16 \%$ (para el maíz en el período 4) (Tabla 4), y todas las diferencias estuvieron a 
favor del MET 4, con los puntos ubicados por encima del valor cero en el eje de las abscisas (Figura 1).

El TND obtenido por el procedimiento de Tilley y Terry (9) (MET 4) para el heno de Tifton (Cynodon dactylon L. Pers. CV. Tifton 85), el maíz y la torta de soya correspondió a 48.44, 92.54 y $87.92 \%$. Para estos mismos alimentos, la determinación mediante modelo multicompartimental (4) (MET 5) derivó cifras de 56.31, 86.99 y $76.62 \%$ TND, respectivamente (Tabla 4 ). Al comparar estos valores con algunos reportes de la literatura, se concluye que el MET 4 subvaloró el contenido energético del heno y sobrestimó el del maíz, en tanto que las predicciones realizadas por el MET 5 estuvieron más afines. El TND reportado por Valadares et al (24) para el heno de Tifton es $55.62 \%$, valor próximo al indicado por el NRC (4), $55.30 \%$, y al rango propuesto por Júnior et al (25), entre 55.90 y $59.61 \%$. Para el maíz, Valadares et al (24), el NRC (4) y Preston (26) reportan $87.24,88.70$ y $88.00 \%$ TND, respectivamente. En relación con la torta de soya, valores sugeridos por Pereira et al (27), Valadares et al (24), Júnior et al (14) y Preston (26), correspondientes a $81.40,81.54,83.24$ y $84.00 \%$ TND, se muestran inferiores al obtenido con MET 4 pero superiores al estimado por MET 5 . No obstante, el NRC (4) y el AEC (28) reportan valores de 88.50 y $77 \%$ TND, respectivamente, muy próximos a los obtenidos con los métodos enen consideración.

Factores como la variabilidad del inóculo, el tamaño de partícula, el peso del sustrato, la relación inóculo:buffer y la duración asignada a la primera etapa del procedimiento pueden explicar los resultados de digestibilidad obtenidos con la técnica de Tilley y Terry (9) en este trabajo, respecto los valores descritos por la literatura.
Boila et al (29) trabajando con un mismo material (ensilaje de maíz) encontraron que la digestibilidad de la materia seca varió en función del peso del sustrato, la frecuencia de agitación de los sustratos durante la incubación, el volumen de inóculo y el número de bacterias presentes en el mismo. Resulta de interés la acertada estimación realizada por el modelo multicompartimental del NRC (4) aun tratándose de alimentos de origen tropical que, como el heno de Tifton (Cynodon dactylon L. Pers. Cv. Tifton 85), presentan contenidos de carbohidratos estructurales. Esto posiblemente se explique por la introducción en el modelo de un término que da cuenta de la proporción de FDN indisponible, donde el contenido de lignina, como factor limitante de la digestión, es considerado. De acuerdo con Weiss (5), la fibra se destaca como la variable más común para predecir el contenido energético de los alimentos, reportando además que la FDN potencialmente digestible calculada en el modelo funciona como una fracción uniforme, o lo que es lo mismo, presenta digestibilidad constante a través de todos los alimentos, lo cual es una característica positiva.

En conclusión, la divergencia en las medidas de dispersión entre los métodos de estimación in vivo e in vitro conduce a recomendar los primeros como el método de elección para la evaluación de alimentos, no obstante, se resalta que el modelo multicompartimental sugerido por el NRC (4) y basado en la descripción química se mostró promisorio para el cálculo del TND.

\section{Agradecimientos}

Los autores agradecen al Proyecto de Sostenibilidad 2011-2012 (CODI, Universidad de Antioquia) y a la Fundación Universitaria San Martín el apoyo económico para la ejecución de este trabajo.

\section{REFERENCIAS}

1. Pond WG, Church DC, Pond KR. Fundamentos de Nutrición y Alimentación de Animales. 2 ed. México: Limusa SA; 2002.

2. Lachmann M, Febres O. La estimación de la digestibilidad en ensayos con rumiantes. [en línea] [fecha de acceso 1 de abril de 2012]. URL disponible en: http:// www.avpa.ula.ve/docuPDFs/xcongreso/ Digestibilidaderumiantes.pdf

3. Alcalde CR, Machado RM, Santos GT, Picolli $\mathrm{R}$, Jobim CC. Digestibilidade in vitro de alimentos com inóculos de líquido de rúmen ou de fezes de bovinos. Acta Scientiarum 2001; 23: 917-921.
4. National Research Council - NRC. Nutrient requirements of dairy cattle. 7 ed. Washington, D.C: National Academy Press; 2001.

5. Weiss WP. Symposium: Prevailing concepts in energy utilization by ruminants. J Dairy Sci 1993; 76: 1802-1811.

6. Brautigan IM. Nutrición Animal. 1 ed. Costa Rica: Universidad Estatal a Distancia; 2007.

7. Inzunza JC. Clasificación de los climas de Köppen. Ciencia Ahora 2005; 15: 1-14. 
8. Valadares Filho SC, Rodrigues P, Magalhães $K$. Exigências nutricionais de zebuínos e tabelas de composição de alimentos. 1 ed. Viçosa: Universidad Federal de Viçosa; 2006.

9. Tilley JMA, Terry RA. A two stage technique for the in vitro digestion of forage crops. J Br Grassl Soc 1963; 18: 104-111.

10. $A N K O M{ }^{\circledR}$ Technology. in vitro true digestibility using the DAISY incubator. Analytical Procedures. Fairport, NY [en línea] [fecha de acceso 20 de abril de 2011]. URL disponible en: http://www.ankom.com/ media/documents/IVDMD_0805_D200.pdf

11. Association of Official Analytical Chemist AOAC. Official Methods of Analysis. 18 ed. Gaithersburg, M.D: AOAC Int.; 2011.

12. Cardona MG, Ayala SA. Protocolos para el laboratorio de Nutrición Animal. Medellín: Universidad de Antioquia; 2004.

13. Van Soest PJ, Robertson JB, Lewis BA. Methods for dietary fiber, neutral detergent fiber and nonstarch polysaccharides in relation to animal nutrition. J Dairy Sci $1991 ; 74: 3583-3597$.

14. Júnior VRR, Valadares Filho SC, Borges AM, Magalhães KA, Ferreira CCB, Valadares RFD, Paulino MF. Determinação do Valor Energético de Alimentos para Ruminantes pelo Sistema de Equações. R Bras Zootec 2003; 32: 473-479.

15. Sniffen CJ, O'Connor JD, Van Soest PJ, Fox DG, Russell JB. A net carbohydrate and protein system for evaluating cattle diets: II. Carbohydrate an protein availability. J Anim Sci 1992; 70: 3562-3577.

16. Van Soest PJ, Wine RH. Use of Detergents in the Analysis of Fibrous Feed. IV. The Determination of Plant Cell Wall Constituents. J Assoc Off Anal Chem 1967; 50: 50-55.

17. Lofgreen GP. The use of digestible energy in the evaluation of feeds. J Anim Sci 1951; 10: $344-351$.

18. Lofgreen GP. The estimation or total digestible nutrients from digestible organic matter. J Anim Sci 1953; 12: 359-365.

19. Crampton EW, Lloyd LE, Mackay VG. The calorie value of TND. J Anim Sci 1957; 16: 541-545.
20. Bland JM, Altman DG. Statistical methods for assessing agreement between two methods of clinical measurement: The analysis of method comparison studies [en línea] 1986 [fecha de acceso 20 de septiembre de 2011]. URL disponible en: http://www-users.york.ac.uk/ mb55/ meas/ba.pdf

21. SAS/STAT - User's Guide (Relase 8.0), Cary, NC, USA: SAS Inst Inc.; 2001.

22. Barth KM, Vander Noot GW, Cason JL. The quantitative relationship between total digestible nutrients and digestible energy values of forages. J Anim Sci 1959; 18: 690-693.

23. National Research Council - NRC. Nutrient requirements of beef cattle. 7 ed. Update. Washington, D.C: National Academy Press; 2000.

24. Valadares Filho SC, Magalhães KA, Rocha Júnior VR, Cappelle ER. Tabelas Brasileiras de Composição de Alimentos Para Bovinos. 1 ed. Viçosa: Universidad Federal de Viçosa; 2006.

25. Júnior JRA, Pereira OG, Garcia R, Valadares Filho SC, Cecon PR, Freitas EVV. Valor Nutritivo do Feno de Capim-tifton 85 (Cynodon spp.) em Diferentes Idades de Rebrota, em Ovinos. R Bras Zootec 2000; 29:2193-2199.

26. Preston RL. Typical composition of commonly used feeds for sheep and cattle. Feed Composition Guide [en línea] 2002 [fecha de acceso 30 de marzo de 2012]. URL disponible en: http://images.beef-mag. com/files/13/feedcomp2002.pdf

27. Pereira EMO, Ezequiel JM, Biagioli B, Feitosa J. Determinação In Vitro do Potencial de Produção de Metano e Dióxido de Carbono de Líquido Ruminal Proveniente de Bovinos de Diferentes Categorias. Arch Latinoam Prod Anim 2006; 14: 120-127.

28. Tablas AEC. Recomendaciones para la Nutrición Animal. 5 ed. Paris: Editorial Rhône Poulenc; 1987.

29. Boila RJ, Erfle JD, Sauer FD. Evaluation on the two-stage tecnique for the in Vitro estimation of the dry matter digestibility of corn silage. Can J Anim Sci 1980; 50: 367-378. 